\title{
Resonance Raman scattering in semiconductor quantum dots: Adiabatic vs. time-dependent perturbation theory
}

\author{
E. Menéndez-Proupin 7 and Nana Cabo-Bisset \\ IMRE-Facultad de Física, Universidad de La Habana, Vedado \\ 10400, La Habana, Cuba
}

(Dated: October 22, 2018)

\begin{abstract}
The adiabatic theory of resonance one-phonon Raman scattering in semiconductor nanocrystals is revised and extended with perturbative non-adiabatic corrections, given by the Albrecht's B term. This theory is confronted with the time-dependent perturbation approach, pointing at their differences and similarities. It is shown that both theories are equivalent in the limit of weak electron-phonon coupling and non-degenerate or uncoupled resonant states. Evaluations of the $\mathrm{A}$ and $\mathrm{B}$ terms for the confined LO phonon in CdSe and CdS nanocrystals are reported. These evaluations show that the B term can usually be neglected.
\end{abstract}

\section{INTRODUCTION}

In the interpretation of resonant Raman scattering in quantum dots, two theories have been mostly used. The first one is based on the Albrecht's theoryt of light scattering from small molecules, where the excited levels that contribute to the Raman polarizability are considered as vibron states in the adiabatic approximation. This theory also considered perturbative non-adiabatic corrections, but these later have been neglected in quantum dot studieste. coupling factor has been identified with the Huang-Rhys factor, using this as a fitting parameter for the relative intensities of different orders in Raman spectra. However, the calculations of the Huang-Rhys-narameter for intrinsic exciton states in $\mathrm{PbS}$ and $\mathrm{CdSe} 10.11$ nanocrystals have given values that are too small compared with those needed to explain the experimental results of multiphonon Raman scattering. Extrinsic mechanisms such as doner like exciton 12 , surface hole trap 13 or extra charges 10.14 have been invoked to resolve the discrepancy

A different model of Raman scattering in quantum dots was conceived from a solid state point of view. In this approach, the Raman cross sections are calculated from third or higher order time-dependent perturbation theory (TDPT) 15.11.17 and the intermediate virtual states are considered as tensor products of electronic states, lattice vibrations, and photons. A non-perturbative calculation of multimhonon Raman spectra have been recently presented 14. Not having adjustable parameters this model has scarcely been used by experimentalists to interpret their data. Moreover, up to now it is unclear the relation between the TDPT and the Albrecht's theory.

The purpose of the present article is: (1) to establish the relation between Albrecht's theory and TDPT, and (2) to investigate the importance of non-adiabatic corrections within Albrecht's theory. The structure of the paper is as fallows. First, we give an overview of both theoretical approaches and show their interrelation. Next, we explain the calculation of the Albrecht's A and B terms for semiconductor nanocrystals. Finally we discuss the numerical results obtained for several types of nanocrys- tals and present our conclusions. Several mathematical steps are given in the appendixes.

\section{THE THEORIES}

\section{A. Time-dependent perturbation theory}

For a one-phonon Raman process the differential cross section is given by 15

$$
\frac{d^{2} \sigma}{d \Omega_{s} d \omega_{s}}=\frac{V^{2} \omega_{s}^{3} \eta_{l} \eta_{s}^{3}}{4 \pi^{2} c^{4} \omega_{l} \hbar} \sum_{F}\left|M_{F I}^{(1)}(p)\right|^{2} \delta\left(\hbar \omega_{l}-\hbar \omega_{s}-\hbar \omega_{p}\right),
$$

where $\eta_{l}\left(\eta_{s}\right)$ is the refraction index at the incident (scattered) light frequency $\omega_{l}\left(\omega_{s}\right), V$ is a normalization volume of the radiation field, and $c$ is the velocity of light in vacuum. The one-phonon transition amplitude $M_{F I}^{(1)}(p)$ can be calculated by time dependent perturbation theory, considering the unperturbed Hamiltonian as the sum of the Electronic, Lattice, and Radiation operators

$$
H_{0}=H_{E}+H_{L}+H_{R},
$$

while the perturbation is the sum of Electron-Lattice, Electron-Radiation, and Lattice-Radiation interactions (the last one is negligible in resonance conditions)

$$
H_{\text {int }}=H_{E-L}+H_{E-R}+H_{L-R} .
$$

The electron-lattice interaction can be expressed as

$$
\begin{aligned}
H_{E-L} & =\sum_{\mu, \mu^{\prime}, \nu}\left\langle\mu\left|H_{E-L}^{+}(\nu)\right| \mu^{\prime}\right\rangle \hat{D}_{\mu}^{\dagger} \hat{D}_{\mu^{\prime}} \hat{b}_{\nu}^{\dagger} \\
& +\sum_{\mu, \mu^{\prime}, \nu}\left\langle\mu\left|H_{E-L}^{-}(\nu)\right| \mu^{\prime}\right\rangle \hat{D}_{\mu}^{\dagger} \hat{D}_{\mu^{\prime}} \hat{b}_{\nu}
\end{aligned}
$$

where $\hat{D}_{\mu^{\prime}}\left(\hat{D}_{\mu}^{\dagger}\right)$ and $\hat{b}_{\nu}\left(\hat{b}_{\nu}^{\dagger}\right)$ are annihilation (creation) operators of electronic and vibrational excitations, respectively, while $H_{E-L}^{+}(\nu)$ and its Hermitian adjoint $H_{E-L}^{-}(\nu)$ are operators that act on the electronic 
system 16 . The operators $H_{E-R}$ and $H_{R-L}$ have a similar structure to that of $H_{E-L}$. The phonon states created by $\hat{b}_{\nu}^{\dagger}$ are those of the electronic ground state $G$. The electronic excitations $\mu \neq G$ can be considered as electronhole pairs and confined excitons. Hence, the one-phonon Raman transition amplitude can be calculated by third order perturbation theory as

$$
\begin{aligned}
M_{F I}^{(1)}(p)= & \sum_{\mu_{1}, \mu_{2}(\neq G)} \frac{\left\langle G\left|H_{E-R}^{+}\right| \mu_{2}\right\rangle}{\left(\hbar \omega_{l}-E_{\mu_{2}}-\hbar \omega_{p}+i \Gamma_{\mu_{2}}\right)} \\
& \times \frac{\left\langle\mu_{2}\left|H_{E-L}^{+}(p)\right| \mu_{1}\right\rangle\left\langle\mu_{1}\left|H_{E-R}^{-}\right| G\right\rangle}{\left(\hbar \omega_{l}-E_{\mu_{1}}+i \Gamma_{\mu_{1}}\right)},
\end{aligned}
$$

where $\Gamma_{\mu}$ are the lifetime broadenings of the electronic excitations. The interaction matrix elements $\left\langle\mu_{2}\left|H_{E-L}^{+}(p)\right| \mu_{1}\right\rangle,\left\langle G\left|H_{E-R}^{+}\right| \mu_{2}\right\rangle$ and $\left\langle\mu_{1}\left|H_{E-R}^{-}\right| G\right\rangle$ have been calculated under two approximations of solid state theory: the Effective Mass Approximation and a Long Wave Continuous Model for the optical phonons (a field theory appraach) Werking expressions can be

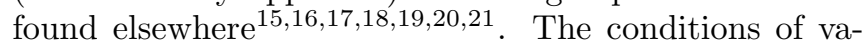
lidity of the above formalism can be summarized as: (1) The scattering process is dominated by extended vibrational states, which are not affected by single-electron excitations, as is usual in large molecules and solids; (2) Excited electronic states are well separated in energy from the ground state.

\section{B. Albrecht's theory}

In this scheme, the wave functions of the molecule (or the quantum dot) are considered in the adiabatic Born-
Oppenheimer approximation

$$
\Psi_{e v}(\{r\},\{Q\})=\Theta_{e}(\{r\},\{Q\}) \Phi_{e v}(\{Q\})
$$

where $e$ and $v$ are the sets of electronic and vibrational quantum numbers, respectively; $\{r\}$ is the ensemble of electron coordinates of the molecule (or the quantum dot), and $\{Q\}$ is the ensemble of normal coordinates of the ions. The vibrational wave function $\Phi_{e v}$ is factored as a product of the wave functions of all the normal modes

$$
\Phi_{e v}(\{Q\})=\varphi_{v_{1}}^{e}\left(Q_{1}\right) \varphi_{v_{2}}^{e}\left(Q_{2}\right) \ldots
$$

$v_{a}$ being phonon occupation numbers. According to the dispersion theory, Albrecht obtained the Raman polarizability tensor for resonance scattering as

$$
\overleftrightarrow{\boldsymbol{\alpha}}=\overleftrightarrow{\boldsymbol{A}}^{\prime \prime \prime}+\overleftrightarrow{B}^{\prime \prime \prime}+\text { h.o.t. }+ \text { n.r.t. }
$$

where h.o.t. means higher order terms and n.r.t. nonresonant terms.

The $\mathrm{A}$ and $\mathrm{B}$ terms are given by

$$
\left(\stackrel{\leftrightarrow}{\boldsymbol{A}}^{\prime \prime \prime}\right)_{g i, g j}=\sum_{(e), v} \mathfrak{M}_{e, g}^{0} \mathfrak{M}_{g, e}^{0} \frac{(g i \mid e v)(e v \mid g j)}{\hbar \omega_{e v, g i}-\hbar \omega_{l}+i \Gamma_{e}^{\prime}}
$$

and

$$
\left(\overleftrightarrow{\boldsymbol{B}}^{\prime \prime \prime}\right)_{g i, g j}=\sum_{(e), v, s, a} \frac{h_{s e}^{a} \mathfrak{M}_{e, g}^{0} \mathfrak{M}_{g, s}^{0}\left(g i\left|Q_{a}\right| e v\right)(e v \mid g j)+h_{e s}^{a} \mathfrak{M}_{s, g}^{0} \mathfrak{M}_{g, e}^{0}(g i \mid e v)\left(e v\left|Q_{a}\right| g j\right)}{\left(\hbar \omega_{e v, g i}-\hbar \omega_{l}+i \Gamma_{e}^{\prime}\right)\left(\hbar \omega_{e, s}\right)}
$$

In the above expression we follow Albrecht's notation 1 , but we use dyadic notation instead of tensor subscripts, $\hbar \omega_{l}$ instead of $h \nu_{0}$ for incoming photon energy, and $\left.\left.\left.\hbar \omega_{\alpha, \beta}=E_{\alpha}-E_{\beta} . \mid g i\right), \mid e v\right), \mid g j\right)$ are the initial, intermediate and final vibrational states (Eq. (4) ), respectively, in the potential energy fields of the electronic states $g$ (ground) and $e$ (excited). $\mathfrak{M}_{\alpha, \beta}^{0}$ and $h_{s e}^{a}$ are, respectively, dipole and electron-phonon interaction matrix elements (see Table $\mathbb{1}$ ). Also, the order of some matrix element indexes is reversed in order to generalize the Albrecht's expressions for the case of complex wave functions. Note that the order of subscripts in matrix elements in this notation is the opposite of that in Dirac's notation. The summation on the index $(e)$ can be restricted to the reso- nant state $e$. The $s$ states in (6) appear from a perturbative expansion of $\Theta_{e}(\{r\},\{Q\})$ in terms of $\Theta_{s}(\{r\},\{0\})$.

The above formalism is valid if: (1) The $e$ states are non-degenerate or uncoupled to other states with the same energy, and (2) Excited electronic states are well separated in energy from the ground state.

\section{The offset oscillators model}

In this model, the vibrational states $\varphi_{i_{a}}^{g}\left(Q_{a}\right)$ and $\varphi_{v_{a}}^{e}\left(Q_{a}\right)$ are assumed to be localized in parabolic $V_{g(e)}\left(Q_{a}\right)$ potentials with the same curvature, but the 
TABLE I: Equivalence of Albrecht and TDPT notations.

\begin{tabular}{lcc}
\hline \hline Magnitude & Albrecht's & TDPT \\
\hline $\begin{array}{l}\text { Electronic } \\
\text { excited states }\end{array}$ & $e, s$ & $\mu_{2}, \mu_{1}$ \\
Electronic & & $G$ \\
ground state & & $G$ \\
Matrix element & $-i \sqrt{\frac{2 \pi \hbar \omega_{s, g}}{V \eta_{l}^{2}}} \mathbf{e}_{l} \cdot \mathfrak{M}_{g, s}^{0}$ & $\left\langle\mu_{1}\left|H_{E-R}^{-}\left(\mathbf{e}_{l}, \mathbf{0}\right)\right| G\right\rangle$ \\
Matrix element & $x_{0} h_{s e}^{p}$ & $\left\langle\mu_{2}\left|H_{E-L}(p)\right| \mu_{1}\right\rangle$ \\
\hline \hline
\end{tabular}

origin of $V_{e}$ shifted in $\Delta Q_{a}=\sqrt{2} x_{a 0} \Delta_{a}=\sqrt{2} \sqrt{\hbar / \omega_{a}} \Delta_{a}$. In many cases, only one vibrational mode is assumed and $\Delta_{a}$ is employed as fitting parameter Moreover, $\Delta_{a}^{2}$ is identified as the Huang-Rhys factor 10 . The integrals appearing in the $\mathrm{A}$ and $\mathrm{B}$ terms are given by

$$
(g l \mid e v)=\sqrt{\frac{v !}{l !}} e^{-\Delta_{a}^{2} / 2} \Delta_{a}^{l-v} L_{v}^{l-v}\left(\Delta_{a}^{2}\right),
$$

and

$$
\begin{aligned}
\left(e v\left|Q_{a}\right| g l\right)= & x_{a 0} \sqrt{\frac{l+1}{2}}(g, l+1 \mid e v)+ \\
& +x_{a 0} \sqrt{\frac{l}{2}}(g, l-1 \mid e v) \\
= & x_{a 0} \frac{l-v+\Delta_{a}^{2}}{\sqrt{2} \Delta_{a}}(g l \mid e v),
\end{aligned}
$$

where $L_{m}^{p}$ are the Laguerre generalized polynomials and $x_{a 0}=\sqrt{\hbar / \omega_{a}}$.

Several authors have used this model tostudy the electron-phonon coupling in nanocrystals 25 , 6. 60 , 8. All of them have considered only the term $\mathrm{A}$ and have used $\Delta_{a}^{2}$ to fit the overtone/fundamental intensity ratios of
Raman spectra. The fitted values of $\Delta_{a}^{2}$ are near 1 , in contradiction with microscopic calculations 10.11 .

\section{The limit of weak electron-phonon coupling}

In the limit of weak electron-phonon coupling the oscillator offset $\Delta_{a}$ should be small. Expanding (7) and (8) in powers of $\Delta_{a}$ we find

$$
(e v \mid g i)=\delta_{v, i}+\left[\sqrt{\frac{i !}{v !}} \delta_{v, i-1}-\sqrt{\frac{v !}{i !}} \delta_{v, i+1}\right] \Delta_{a}+O\left(\Delta_{a}^{2}\right)
$$

and

$$
\begin{aligned}
& \left(e v\left|Q_{a}\right| g i\right)=\int \varphi_{i_{a}}\left(Q_{a}\right) \varphi_{v_{a}}\left(Q_{a}\right) Q_{a} d Q_{a} \\
& =x_{a 0}\left[\sqrt{\frac{v_{a}}{2}} \delta_{v_{a}, i_{a}+1}+\sqrt{\frac{i_{a}}{2}} \delta_{v_{a}, i_{a}-1}\right]+\frac{x_{0 a} \Delta_{a}}{\sqrt{2}} \\
& \quad \times\left[\sqrt{\frac{i !}{v !}}\left((i+1) \delta_{v, i}+\delta_{v, i-2}\right)-\sqrt{\frac{v !}{i !}}\left(i \delta_{v, i}+\delta_{v, i+2}\right)\right] \\
& +O\left(\Delta_{q}^{2}\right) .
\end{aligned}
$$

Replacing (9) in Eq. (5) we obtain

$$
\left(\overleftrightarrow{\boldsymbol{A}}^{\prime \prime \prime}\right)_{g i, g, j}=\delta_{i, j} \frac{\mathfrak{M}_{e, g}^{0} \mathfrak{M}_{g, e}^{0}}{E_{e, g}-\hbar \omega_{l}-\Delta_{a}^{2} \hbar \omega_{a}+i \Gamma_{e}^{\prime}}+O\left(\Delta_{a}\right)
$$

This means that $\overleftrightarrow{\boldsymbol{A}}^{\prime \prime \prime}$ contributes mainly to Rayleigh scattering. Nevertheless, the term proportional to $\Delta_{a}$ is important for one-phonon Raman scattering. To first order in $\Delta_{a}$ we find that

$$
\left(\overleftrightarrow{\boldsymbol{A}}^{\prime \prime \prime}\right)_{g 0_{a}, g 1_{a}}=\frac{-\mathfrak{M}_{e, g}^{0} \mathfrak{M}_{g, e}^{0} \Delta_{a} \hbar \omega_{a}}{\left(E_{e, g}-\Delta_{a}^{2} \hbar \omega_{a}-\hbar \omega_{l}+i \Gamma_{e}^{\prime}\right)\left(E_{e, g}+\left(1-\Delta_{a}^{2}\right) \hbar \omega_{a}-\hbar \omega_{l}+i \Gamma_{e}^{\prime}\right)} .
$$

On the other hand, substituting (9) and (10) in (6) we obtain that, up to first order in the electron-phonon interaction, the B term is non-null only for one-phonon Raman processes. For Stokes processes at low temperature $\left(i_{a}=0, j_{a}=1\right)$ we obtain

$$
\left(\stackrel{\leftrightarrow}{\boldsymbol{B}}^{\prime \prime \prime}\right)_{g 0_{a}, g 1_{a}}=\sum_{(e), s}\left\{\frac{\mathfrak{M}_{e, g}^{0} \mathfrak{M}_{g, s}^{0} h_{s e}^{a} x_{a 0} / \sqrt{2}}{\left(E_{e, g}+\left(1-\Delta_{a}^{2}\right) \hbar \omega_{a}-\hbar \omega_{l}+i \Gamma_{e}^{\prime}\right)\left(E_{e}-E_{s}\right)}+\frac{\mathfrak{M}_{s, g}^{0} \mathfrak{M}_{g, e}^{0} h_{e s}^{a} x_{a 0} / \sqrt{2}}{\left(E_{e, g}-\Delta_{a}^{2} \hbar \omega_{a}-\hbar \omega_{l}+i \Gamma_{e}^{\prime}\right)\left(E_{e}-E_{s}\right)}\right\}
$$

In the second term, one can exchange the indexes $e$ and $s$. Next, under the condition $\left|E_{e}-E_{s}\right| \gg\left|\hbar \omega_{a}+i\left(\Gamma_{e}^{\prime}-\Gamma_{s}^{\prime}\right)\right|$ one obtains

$$
\left(\stackrel{\leftrightarrow}{\boldsymbol{B}}^{\prime \prime \prime}\right)_{g 0_{a}, g 1_{a}}=\sum_{e, s \neq e} \frac{-\mathfrak{M}_{e, g}^{0} \mathfrak{M}_{g, s}^{0} h_{s e}^{a} x_{a 0} / \sqrt{2}}{\left(E_{e, g}+\left(1-\Delta_{a}^{2}\right) \hbar \omega_{a}-\hbar \omega_{l}+i \Gamma_{e}^{\prime}\right)\left(E_{s, g}-\Delta_{a}^{2} \hbar \omega_{a}-\hbar \omega_{l}+i \Gamma_{s}^{\prime}\right)}
$$

Noting that $\Delta_{a}^{2} \ll 1, \Delta_{a} \hbar \omega_{a}=-\left\langle e\left|H_{E-L}(a)\right| e\right\rangle$, and $h_{s e}^{a} x_{a 0} / \sqrt{2}=\left\langle e\left|H_{E-L}(a)\right| s\right\rangle$ (see Appendixes A and B), we see that $\overleftrightarrow{\boldsymbol{A}}^{\prime \prime \prime}+\overleftrightarrow{\boldsymbol{B}}^{\prime \prime \prime}$ for one-phonon emission reduces 
TABLE II: Parameters used in the calculations When not indicated, the source is Ref. 20 for CdS and Ref. 22 for CdSe.

\begin{tabular}{llll}
\hline \hline Parameter & CdS & CdSe & CdSe (MBEMA) \\
\hline$E_{g}(\mathrm{eV})$ & 2.6 & 1.865 & $1.841^{a}$ \\
$m_{e} / m_{0}$ & 0.18 & 0.12 & $0.13^{b}$ \\
$m_{h} / m_{0}$ & 0.51 & 0.45 & \\
$\gamma_{1}$ & & & $1.66^{b}$ \\
$\gamma_{2}$ & & & $0.41^{b}$ \\
$2 m_{0} P^{2}(\mathrm{eV})$ & $21^{c}$ & $20^{c}$ & $20^{c}$ \\
$\kappa$ & 7.8 & 9.53 & 9.53 \\
$V_{e}(\mathrm{eV})$ & 2.5 & $\infty$ & $0.6^{b}$ \\
$V_{h}(\mathrm{eV})$ & 1.9 & $\infty$ & $\infty^{b}$ \\
$\omega_{L}\left(\mathrm{~cm}^{-1}\right)$ & 305 & 213 & 213 \\
$\omega_{T}\left(\mathrm{~cm}^{-1}\right)$ & 238 & 165 & 165 \\
$\epsilon_{0}$ & $8.7^{d}$ & 9.53 & 9.53 \\
$\epsilon_{\infty}$ & 5.3 & $5.72^{d}$ & $5.72^{d}$ \\
$\beta_{L}\left(10^{-6}\right)$ & 2.68 & 1.58 & 1.58 \\
$\Gamma_{\mu}\left(\mathrm{meV}^{2}\right)$ & 5 & 5 & 5 \\
\hline \hline
\end{tabular}

${ }^{a}$ Ref. 23.

${ }^{b}$ Ref. 24

${ }^{c}$ Ref. 25.

${ }^{d}$ Calculated from the Lydanne-Sachs-Teller relation.

to the same result that the TDPT.

\section{CALCULATION OF $\overleftrightarrow{A}^{\prime \prime \prime}$ AND $\overleftrightarrow{B}^{\prime \prime \prime}$}

\section{A. Effective Mass Approximation}

We use the exciton wave functions and the electronphonon operator of Ref. 20 to estimate the A and B terms in nanocrystals of several semiconductors. Using the Table If and the Effective Mass Approximation we have

$$
\mathfrak{M}_{g, e}^{0}=\frac{i e}{m_{0} \omega_{e, g}} \mathbf{p}_{s_{z} j_{z} ; e} f_{o_{e}}
$$

where $f_{o_{e}}$ is the envelope overlap integral ( $o_{e}$ being the set of envelope quantum numbers)

$$
f_{o_{e}}=\int \Psi_{o_{e}}(\mathbf{r}, \mathbf{r})^{*} d^{3} \mathbf{r}
$$

$\mathbf{p}_{s_{z} J_{z} ; e}$ is the bulk momentum matrix element between the couple of bands to which the exciton $e$ belongs. For the valence band $J_{z}= \pm 3 / 2, \pm 1 / 2$ and for the conduction band $s_{z}= \pm 1 / 2$.

We evaluate the relative importance of the terms $\mathrm{A}$ and B for semiconductor nanocrystals. Assuming only one vibrational mode and focusing on one-phonon creation processes at low temperature (i. e. $i=0$ and $j=1$ ), the resonant term in (5) is reduced to

$$
\begin{aligned}
\left(\overleftrightarrow{\boldsymbol{A}}^{\prime \prime \prime}\right)_{g i, g j} & =\left(\sum_{s_{z}, J_{z}} \mathbf{p}_{s_{z} J_{z} ; e}^{*} \mathbf{p}_{s_{z} J_{z} ; e}\right)\left(\frac{e \hbar f_{o_{e}}}{m_{0} E_{e, g}}\right)^{2} \\
& \times \sum_{v} \frac{(g i \mid e v)(e v \mid g j)}{E_{e, g}+\left(v-\Delta^{2}\right) \hbar \omega_{L O}-\hbar \omega_{l}+i \Gamma_{e}^{\prime}}
\end{aligned}
$$

The first term between parentheses is a band factor and is the responsible of the angular pattern of the scattered intensity. The summation over $s_{z}, J_{z}$, is performed to take into account the degeneracy of conduction and hole bands, giving 26

$$
\sum_{s_{z}, J_{z}} \mathbf{p}_{s_{z} J_{z} ; e}^{*} \mathbf{p}_{s_{z} J_{z} ; e}=\frac{(2 J+1)}{3}\left(m_{0} P\right)^{2} \overleftrightarrow{\mathbf{1}}
$$

where $J=3 / 2(1 / 2)$ if the upper valence band have $\Gamma_{8}$ $\left(\Gamma_{7}\right)$ symmetry and $P=-i\left\langle S\left|\hat{p}_{x}\right| X\right\rangle / m_{0}$.

To evaluate the $B$ term we also need to include the electron-phonon matrix elements $x_{0} h_{s e}=$ $\sqrt{2}\left\langle e\left|H_{E-L}\right| s\right\rangle$ (see Appendix B). With these considerations we obtain

$$
\begin{aligned}
& \left(\overleftrightarrow{\boldsymbol{B}}^{\prime \prime \prime}\right)_{g i, g j}=\left(\sum_{s_{z} J_{z}} \mathbf{p}_{s_{z} J_{z} ; e} \mathbf{p}_{s_{z} J_{z} ; s}\right)\left(\frac{e \hbar}{m_{0}}\right)^{2} \\
& \times \sum_{v, o s} \frac{f_{o e} f_{o s}\left\langle e\left|H_{E-L}\right| s\right\rangle}{E_{e, g} E_{s, g} E_{e, s}} \frac{\sqrt{2}}{x_{0}} \\
& \quad \times \frac{(g i|Q| e v)(e v \mid g j)+(g i \mid e v)(e v|Q| g j)}{E_{e, g}+\left(v-\Delta^{2}\right) \hbar \omega_{L O}-\hbar \omega_{l}+i \Gamma_{e}^{\prime}}
\end{aligned}
$$

We evaluate $\left\langle e\left|H_{E-L}\right| s\right\rangle$ as in Ref. 20. Due to the Fröhlich interaction cannot cause intersubband transitions, $\mathbf{p}_{s_{z} J_{z} ; s}^{*}=\mathbf{p}_{s_{z} J_{z} ; e}^{*}$ and the band factor in (14) is the same as that in (12). Intersubband transitions may occur via deformation potential interaction, but these are usually negligible in polar materials. It must be noticed that it is not possible to consider this mechanism within the Albrecht's theory, as the existence of degenerate hole states connected by the electron-phonon interaction means a breakdown of the adiabatic approximation, which is reflected in null denominators $E_{e, s}$ in Eq. (14). Nevertheless, TDPT can deal with it without trouble. From this result we conclude that the A and B terms are scalars (with small tensor corrections for B) and the integration over nanocrystal orientations and light polarization has no effect on the ratio between the $\mathrm{A}$ and $\mathrm{B}$ terms.

\section{B. Multiband effective mass theory}

In a multiband formalism, the essential effect of band mixing can be captured using the spherical approximation for the hole Hamiltoniane 27

$$
H_{h}=\frac{\gamma_{1}}{2 m_{0}}\left(\hat{\mathbf{p}}^{2}-\frac{\mu}{9}\left(\mathbf{P}^{(2)} \cdot \mathbf{J}^{(2)}\right)\right)+V(r),
$$

$V(r)$ being the confinement potential, $\mathbf{P}^{(2)}$ and $\mathbf{J}^{(2)}$ are spherical rank tensors built from linear and angular momentum operators, $\mu=2 \gamma_{2} / \gamma_{1}$, and $\gamma_{2}$ and $\gamma_{1}$ are Luttinger parameters.

Electron-hole pair states with well defined total (Bloch+orbital) angular momentum quantum numbers 
$M$ and $M_{z}$ can be obtained as

$$
\begin{aligned}
&|e\rangle= \sum_{n, N, l, L, f, F} C_{n N l L s J f F M M_{z}}^{(e)}\left|n N l L s J f F M M_{z}\right\rangle \\
&= \sum_{n, N, l, L, f, F, f_{z}, F_{z}} C_{n N l L s J f F M M_{z}}^{(e)}\left(f F f_{z} F_{z} \mid M M_{z}\right) \\
& \times\left|n l s f f_{z}\right\rangle \otimes\left|N L J F F_{z}\right\rangle,
\end{aligned}
$$

where $\left(f F f_{z} F_{z} \mid M M_{z}\right)$ is a Clebsch-Gordan coefficient. Lowercase (uppercase) letters denote electron (hole) quantum numbers. $\left|n l s f f_{z}\right\rangle$ and $\left|N L J F F_{z}\right\rangle$ are electron and hole states with well defined total angular momentum, their wave functions given by

$$
\left\langle\mathbf{r} \mid n l s f f_{z}\right\rangle=\sum_{l_{z}, s_{z}}\left(l s l_{z} s_{z} \mid f f_{z}\right) R_{n l}(r) Y_{l l_{z}}(\theta, \varphi)\left\langle\mathbf{r} \mid s s_{z}\right\rangle
$$

and

$$
\begin{aligned}
\left\langle\mathbf{r} \mid N L J F F_{z}\right\rangle= & \sum_{K=L, L+2} \sum_{L_{z}, J_{z}}\left(K J L_{z} J_{z} \mid f f_{z}\right) \\
& \times R_{N K}^{(F, L)}(r) Y_{K L_{z}}(\theta, \varphi)\left\langle\mathbf{r} \mid J J_{z}\right\rangle .
\end{aligned}
$$

In the above expression $R_{n l}(r)$ are the radial wave functions of a particle in a spherical box and $R_{N K}^{(F, L)}(r)$ are the solutions of the MBEMA equations given elsewhere 28.29, $Y_{l l_{z}}(\theta, \varphi)$ are the spherical harmonics $30,\left\langle\mathbf{r} \mid s s_{z}\right\rangle$ are $\Gamma_{6}$ Bloch function and $\left\langle\mathbf{r} \mid J J_{z}\right\rangle$ are hole Bloch functions. The hole Bloch functions are related with the $\Gamma_{8}(J=3 / 2)$ electronic Bloch functions $\left|\overline{J, J_{z}}\right\rangle$ by the rule $\left|J J_{z}\right\rangle=$ $(-1)^{J-J_{z}}\left|\overline{J,-J_{z}}\right\rangle$ (derived from the time-reversal operation). Our $\left|J J_{z}\right\rangle$ are $|3 / 2, \pm 3 / 2\rangle=\mp(i / \sqrt{2})(X \pm i Y)| \pm\rangle$, and $|3 / 2, \pm 1 / 2\rangle=(i / \sqrt{6})[2 Z| \pm\rangle \mp(X \pm i Y)|\mp\rangle]$.

Within this basis, in the strong confinement regime, the Coulomb interaction can be treated by direct diagonalization of the Hamiltonian or even by simple pertwr bation theory. Using the theory of angular momentum 31 , compact expressions for the matrix elements can be obtained. The dipole matrix elements gives

$$
\begin{aligned}
& \mathfrak{M}_{g, e}^{0}=i \hat{\mathbf{e}}_{M_{z}}^{*} \frac{2 P e \hbar}{E_{e, g}} \delta_{M, 1}(-1)^{f+5 / 2} \sqrt{\frac{(2 f+1)(2 F+1)}{3}} \\
& \quad \times\left\{\begin{array}{ccc}
1 & 3 / 2 & 1 / 2 \\
l & f & F
\end{array}\right\}\left(\delta_{l, L}+\delta_{l, L+2}\right) \int R_{N l}^{(F, L)}(r) R_{n l}(r) r^{2} d r
\end{aligned}
$$

where $R_{n l}$ and $R_{N L}^{(F, L)}$ are electron and hole radial functions, respectively, and

$$
\hat{\mathbf{e}}_{0}=\mathbf{k}, \quad \hat{\mathbf{e}}_{ \pm 1}=\mp \frac{\mathbf{i} \pm i \mathbf{j}}{\sqrt{2}}
$$

$\mathbf{i}, \mathbf{j}$ and $\mathbf{k}$ being the unit vectors along the $\mathrm{X}-$, $\mathrm{Y}$ - and Z-axis, respectively.

In the A term we must sum over the degenerate $e$ states with different $M_{z}$, which turns out in a term proportional to the diagonal tensor

$$
\begin{aligned}
\left(\overleftrightarrow{\boldsymbol{A}}^{\prime \prime \prime}\right)_{g i, g j}= & \stackrel{\leftrightarrow}{\mathbf{1}} \frac{4 P^{2} e^{2} \hbar^{2}}{E_{e, g}^{2}} \delta_{M, 1}\left(\delta_{l_{e}, L_{e}}+\delta_{l_{e}, L_{e}+2}\right) \frac{\left(2 f_{e}+1\right)\left(2 F_{e}+1\right)}{3}\left\{\begin{array}{ccc}
1 & 3 / 2 & 1 / 2 \\
l_{e} & f_{e} & F_{e}
\end{array}\right\}^{2} \\
& \times\left[\int R_{N_{e} l_{e}}^{\left(F_{e} L_{e}\right)}(r) R_{n_{e} l_{e}}(r) r^{2} d r\right]^{2} \sum_{v} \frac{(g i \mid e v)(e v \mid g i)}{E_{e, g}+\left(v-\Delta^{2}\right) \hbar \omega_{L O}-\hbar \omega_{l}+i \Gamma_{e}^{\prime}}
\end{aligned}
$$

The $\mathrm{B}$ term for $l_{p}=0$ phonons is given by

$$
\begin{aligned}
\left.\overleftrightarrow{\boldsymbol{B}}^{\prime \prime \prime}\right|_{l_{p}=0}= & \overleftrightarrow{\mathbf{1}} \delta_{M, 1} \sum_{(e), s} \frac{4 P^{2} e^{2} \hbar^{2}}{3 E_{e, g} E_{s, g}}\left\{\begin{array}{ccc}
1 & 3 / 2 & 1 / 2 \\
l_{e} & f_{e} & F_{e}
\end{array}\right\}\left\{\begin{array}{ccc}
1 & 3 / 2 & 1 / 2 \\
l_{s} & f_{s} & F_{s}
\end{array}\right\}(-1)^{f_{e}+f_{s}+3} \\
& \times \sqrt{\left(2 f_{e}+1\right)\left(2 f_{s}+1\right)\left(2 F_{e}+1\right)\left(2 F_{s}+1\right)}\left(\delta_{l_{e}, L_{e}}+\delta_{l_{e}, L_{e}+2}\right)\left(\delta_{l_{s}, L_{s}}+\delta_{l_{s}, L_{s}+2}\right) \\
& \times \int R_{N_{e} l_{e}}^{\left(F, L_{e}\right)}(r) R_{n_{e} l_{e}}(r) r^{2} d r \int R_{N_{s} l_{s}}^{\left(F, L_{s}\right)}(r) R_{n_{s} l_{s}}(r) r^{2} d r\left\langle s\left|H_{E-L}^{\left(l_{p}=0\right)}\right| e\right\rangle \\
& \times \sum_{v} \frac{\sqrt{2}}{x_{0}} \frac{(g i|Q| e v)(e v \mid g j)+(g i \mid e v)(e v|Q| g j)}{\left(E_{e, s}\right)\left(E_{e, g}+\left(v-i-\Delta^{2}\right) \hbar \omega_{L O}-\hbar \omega_{l}+i \Gamma_{e}^{\prime}\right)}
\end{aligned}
$$

\section{DISCUSSION}

Table III shows the Huang-Rhys parameter $\Delta^{2}$, the A term absolute value, and the ratio $\left|A^{\prime \prime \prime} / B^{\prime \prime \prime}\right|$, calcu- lated for typical nanocrystals $20 \AA$ in radius. The photon energies, corresponding to incoming resonance with the lower exciton level are also indicated in the table. The incomplete exciton confinement in CdS nanocrystals has 
TABLE III: Numerical results for typical QD's $20 \AA$ in radius. The energies $\hbar \omega_{l}$ correspond to incoming resonance with the lower Raman active exciton level in each nanocrystal.

\begin{tabular}{lllll}
\hline \hline Nanocrystal & $\Delta^{2}$ & $\hbar \omega_{l}(\mathrm{eV})$ & $\left|A^{\prime \prime \prime} / B^{\prime \prime \prime}\right|$ & $\left|A^{\prime \prime \prime}\right|\left(\AA^{3}\right)$ \\
\hline CdS & 0.08 & 2.870 & 13 & $8.1 \times 10^{4}$ \\
CdS $\left(R_{e f}\right)$ & 0.0013 & 2.878 & 8.4 & $1.2 \times 10^{4}$ \\
CdSe & 0.0008 & 2.592 & 27 & $7.9 \times 10^{3}$ \\
CdSe (MBEMA) & 0.2 & 2.280 & 32 & $6.4 \times 10^{4}$ \\
\hline \hline
\end{tabular}

been considered with two different models: (1) Finite band offsets $V_{e}$ and $V_{h}^{21}$, and (2) An effective radius20. Moreover, for CdSe nanocrystals we have also considered two models: (1) Effective radius22 and (2) Multiband effective mass approximation (MBEMA) along with finite conduction band offset $V^{24}$

As it has been noticed20 21, Raman scattering is quasiforbidden in quantum dots in the strong confinement regime. The scattering is possible through a decompensation between the electron and hole wave functions, which may appear due to (1) the Coulomb electron-hole interaction and difference between electron and hole masses, (2) difference in electron and hole confinement, (3) hole band mixing, (4) defects, and (5) non-adiabatic effects 14 . The electron-hole decompensation also has a direct relation with the Huang-Rhys parameter. This one is larger in the case of CdS, where incomplete confinement have been considered, and in CdSe when band mixing and incomplete electron confinement have been included. These ones are the cases where larger Raman polarizabilities are obtained. Notice that, due to the finite electron confinement assumed, our Huang-Rhys parameter for CdSe nanocrystals within MBEMA is larger than other reported theoretical values 10,11 and is yithin the order of magnitude of the experimental valuest3. 32.33 .

In all the cases examined, the A term determines the Raman polarizability. This means that the interpretation of one-phonon Raman cross section considering only the A term is consistent with the microscopic calculations using TDPT reported here and in Refs. 15, 20,21. However, it is incorrect to fit the Huang-Rhys factor from the overtone to fundamental intensity ratios in multiphonon Raman spectra, as different scattering channels give substantial contributions to the overtones $44[6] 17$.

Let us consider the participation of optical phonons with $l_{p}>0$ in Raman scattering. These phonons connect degenerate band-mixed exciton states and breaks the adiabatic approximation. Hence, the Albrecht's theory cannot describe Raman scattering from these phonons. On the other hand, the TDPT, may deal with degenerate states and non-adiabatic processes. Calculations in Ref. 34 indicate that the role of $l_{p}>0$ phonons in one-phonon Raman spectra is to cause a small shoulder near the interface phonon frequency, being unimportant for the principal peak. Nevertheless, the electron-lattice interaction breaks the degeneracy of the exciton states, causing a redistribution of the exciton-phonon energy levels and possibly originate exciton-phonon complexes. Both these factors could substantially alter the predicted Raman spectra. This effect can be considered by TDPT of higher order in the electron-lattice interaction, or by a non-perturbative calculation of the exciton-phonon complexes. Research on this direction is presently in progress.

\section{CONCLUSIONS}

We have established the connection between the two theories more used for resonance Raman scattering in semiconductor nanocrystals: the Albrecht's theory based on the adiabatic approximation and the time dependent perturbative approach. In particular, we have shown that both theories are equivalent in the limit of weak electron-lattice interaction and when the resonant exciton level is non-degenerate or is a set of uncoupled degenerate states. We have evaluated the relative importance of the Albrecht's A and B terms (the last one not discussed in the literature) for CdSe and CdS nanocrystals, using different models for the electronic excitations. We have found that the A term is the leading coefficient in all the cases considered. Additionally, we have given the expressions of the matrix elements of the electron-radiation and the electron-lattice interactions for a model of exciton considering the fourfold degeneracy of the $\Gamma_{8}$ valence band. In the framework of this model, we have obtained a theoretical Huang-Rhys parameter within the order of magnitude of experimental values.

\section{Acknowledgments}

The authors are grateful to Prof. Carlos Trallero-Giner for their useful suggestions and for a critical reading of the manuscript. This work was partially supported by Alma Mater project 26-2000 of Havana University.

\section{APPENDIX A: THE OSCILLATOR OFFSET}

The adiabatic vibrational eigenstates for the electronic state $e$ are obtained from the Hamiltonian

$$
\begin{aligned}
\hat{H}_{v i b r}^{e} & =\left\langle e\left|H_{L}+H_{E-L}\right| e\right\rangle \\
& =\sum_{a}\left\{\hbar \omega_{a} \hat{b}_{a}^{\dagger} \hat{b}_{a}+\left\langle e\left|H_{E-L}(a)\right| e\right\rangle\left(\hat{b}_{a}+\hat{b}_{a}^{\dagger}\right)\right\}
\end{aligned}
$$

We have considered the case of Hermitian $H_{E-L}(a)$. For non-Hermitian $H_{E-L}(a)$ see Appendix B. The unitary transformation $\hat{c}_{a}=\hat{b}_{a}-\alpha_{a}$, with $\alpha_{a}=$ $-\left\langle e\left|H_{E-L}(a)\right| e\right\rangle / \hbar \omega_{a}$ diagonalizes the Hamiltonian

$$
H_{v i b r}^{e}=\sum_{a} \hbar \omega_{a}\left(\hat{c}_{a}^{\dagger} \hat{c}_{a}-\alpha_{a}^{2}\right)
$$


The new and old phonon coordinate operators are related by

$$
Q_{a}^{e}=\sqrt{\frac{\hbar}{2 \omega_{a}}}\left(\hat{c}_{a}+\hat{c}_{a}^{\dagger}\right)=Q_{a}^{G}-\sqrt{2} \sqrt{\frac{\hbar}{\omega_{a}}} \alpha_{a} .
$$

This relation identifies $\alpha_{a}$ with the oscillator offset $\Delta_{a}$.

\section{APPENDIX B: THE CONNECTION BETWEEN THE CLASSICAL AND THE QUANTUM ELECTRON-PHONON INTERACTION}

The Hamiltonian operator determining the electronic eigenstates can be expanded in Taylor series of the vibrational normal coordinates

$$
H_{E}(Q)=H_{E}(0)+\sum_{a} \frac{\partial H_{E}}{\partial Q_{a}} Q_{a}
$$

The second term of the above formula is the electronphonon interaction.

$$
H_{E-L}=\sum_{a} \frac{\partial H_{E}}{\partial Q_{a}} Q_{a}
$$

The flantum Fröhlich-type interaction operator have the form 35

$$
H_{E-L}=\sum_{a} H_{E-L}^{-}(a) \hat{b}_{a}+H_{E-L}^{+}(a) \hat{b}_{a}^{\dagger}
$$

where $\hat{b}_{a}$ are annihilation operator of phonons in the normal modes $a$. $H_{E-L}^{ \pm}(a)$ are operators that act on the electronic coordinates, e.g. $H_{E-L}^{-}(n, l, m)=$ $\Phi_{n, l}(r) Y_{l, m}(\theta, \varphi)$ for the one-electron-phonon interaction in a semiconductor nanocrystal15. Making the transformation to coordinate and momentum operators

$$
\hat{b}_{a}=\sqrt{\frac{\omega_{a}}{2 \hbar}}\left(Q_{a}+\frac{i}{\omega_{a}} P_{a}\right),
$$

the interaction operator becomes

$$
\begin{aligned}
H_{E-L}= & \sum_{a} \sqrt{\frac{\omega_{a}}{2 \hbar}}\left(H_{E-L}^{-}(a)+H_{E-L}^{+}(a)\right) Q_{a} \\
& +\frac{i}{\sqrt{2 \hbar \omega_{a}}}\left(H_{E-L}^{-}(a)-H_{E-L}^{+}(a)\right) P_{a} .
\end{aligned}
$$

If $H_{E-L}^{-}(a)=H_{E-L}^{+}(a)=H_{E-L}(a)$ then $(\mathrm{B} 1)$ and $\mathrm{B} 2$ are equivalent and

$$
h_{e s}^{a}=\left\langle s\left|\frac{\partial H_{e l}}{\partial Q_{a}}\right| e\right\rangle=\sqrt{\frac{2 \omega_{a}}{\hbar}}\left\langle s\left|H_{E-L}(a)\right| e\right\rangle .
$$

When the vibrational modes in a nanostructure are described by complex fields there are modes for which $H_{E-L}^{-}(a) \neq H_{E-L}^{+}(a)$. In this case, thanks to time reversal symmetry, complex modes are double degenerate and real fields can be obtained from the real and imaginary parts of the complex fields, which correspond to real normal coordinates. The new matrix elements can be obtained from the complex matrix elements as

$$
\begin{aligned}
& \left\langle s\left|H_{E-L}^{(1)}(a)\right| e\right\rangle=\frac{\left\langle s\left|H_{E-L}^{-}(a)+H_{E-L}^{+}(a)\right| e\right\rangle_{(B)}}{\sqrt{2}}(\mathrm{~B} 3 \mathrm{a}) \\
& \left\langle s\left|H_{E-L}^{(2)}(a)\right| e\right\rangle=i \frac{\left\langle s\left|H_{E-L}^{-}(a)-H_{E-L}^{+}(a)\right| e\right\rangle_{\mathrm{B}}}{\sqrt{2}}
\end{aligned}
$$

\section{APPENDIX C: EXCITON PHONON MATRIX ELEMENTS WITH DEGENERATE BANDS}

The matrix elements of the interaction of band-mixed excitons with the optical phonons in a spherical nanocrystal can be calculated following the procedure outlined in Ref. 19. We obtained the expression

$$
\begin{aligned}
& \left\langle n^{\prime} N^{\prime} l^{\prime} L^{\prime} s J f^{\prime} F^{\prime} M^{\prime} M_{z}^{\prime}\left|H_{E-L}^{-}\left(n_{p}, l_{p}, m_{p}\right)\right| n N l L s J f F M M_{z}\right\rangle \\
& =\left\langle n N l L s J f F M M_{z}\left|H_{E-L}^{+}\left(n_{p}, l_{p}, m_{p}\right)\right| n^{\prime} N^{\prime} l^{\prime} L^{\prime} s J f^{\prime} F^{\prime} M^{\prime} M_{z}^{\prime}\right\rangle \\
& =(-1)^{M^{\prime}-M_{z}^{\prime}} \frac{C_{F}}{\sqrt{R}}\left(\begin{array}{ccc}
M^{\prime} & l_{p} & M \\
-M_{z}^{\prime} & m_{p} & M_{z}
\end{array}\right) \sqrt{(2 M+1)\left(2 M^{\prime}+1\right)\left(2 l_{p}+1\right) / 4 \pi} \\
& \times\left\{-\delta_{F, F^{\prime}} \delta_{N, N^{\prime}} \delta_{L, L^{\prime}}(-1)^{F+M+f+f^{\prime}+s} \sqrt{\left(2 f^{\prime}+1\right)(2 f+1)\left(2 l^{\prime}+1\right)(2 l+1)}\right. \\
& \times\left\{\begin{array}{ccc}
f^{\prime} & f & l_{p} \\
l & l^{\prime} & s
\end{array}\right\}\left\{\begin{array}{ccc}
M^{\prime} & M & l_{p} \\
f & f^{\prime} & F
\end{array}\right\}\left(\begin{array}{ccc}
l^{\prime} & l_{p} & l \\
0 & 0 & 0
\end{array}\right) \int R_{n^{\prime} l^{\prime}}(r) \Phi_{n_{p}, l_{p}}(r) R_{n l}(r) r^{2} d r \\
& +\delta_{f, f^{\prime}} \delta_{n, n^{\prime}} \delta_{l, l^{\prime}}(-1)^{M^{\prime}+f+J+2 F} \sum_{K=L, L+2} \sum_{K^{\prime}=L^{\prime}, L^{\prime}+2} \sqrt{\left(2 F^{\prime}+1\right)(2 F+1)\left(2 K^{\prime}+1\right)(2 K+1)} \\
& \left.\times\left\{\begin{array}{ccc}
F^{\prime} & F & l_{p} \\
K & K^{\prime} & J
\end{array}\right\}\left\{\begin{array}{ccc}
M^{\prime} & M & l_{p} \\
F & F^{\prime} & f
\end{array}\right\}\left(\begin{array}{ccc}
K^{\prime} & l_{p} & K \\
0 & 0 & 0
\end{array}\right) \int R_{N^{\prime} K^{\prime}}^{\left(F^{\prime}, L^{\prime}\right)}(r) \Phi_{n_{p}, l_{p}}(r) R_{N K}^{(F, L)}(r) r^{2} d r\right\},
\end{aligned}
$$


where $\Phi_{n_{p}, l_{p}}(r)$ is the radial part of the optical phonons electrostatic potential 15 . The optical modes for $l_{p}>0$ are described by complex fields, real field matrix elements can be obtained from Eq. (B3).

For the Coulomb electron-hole interaction we obtained the expression

$$
\begin{aligned}
&\left\langle n^{\prime} N^{\prime} l^{\prime} L^{\prime} s J f^{\prime} F^{\prime} M^{\prime} M_{z}^{\prime}\left|\frac{1}{\left|\mathbf{r}_{e}-\mathbf{r}_{h}\right|}\right| n N l L s J f F M M_{z}\right\rangle=\delta_{M, M^{\prime}} \delta_{M_{z}, M_{z}^{\prime}}(-1)^{s+J+2 f+F+F^{\prime}+M} \\
& \times \sqrt{(2 f+1)\left(2 f^{\prime}+1\right)(2 F+1)\left(2 F^{\prime}+1\right)} \sum_{K, K^{\prime}, p}\left\{\begin{array}{ccc}
p & f & f^{\prime} \\
s & l^{\prime} & l
\end{array}\right\}\left\{\begin{array}{ccc}
p & F & F^{\prime} \\
J & K^{\prime} & K
\end{array}\right\}\left\{\begin{array}{ccc}
p & f & f^{\prime} \\
M & F^{\prime} & F
\end{array}\right\} \\
& \times \sqrt{(2 l+1)\left(2 l^{\prime}+1\right)(2 K+1)\left(2 K^{\prime}+1\right)}\left(\begin{array}{ccc}
l^{\prime} & p & l \\
0 & 0 & 0
\end{array}\right)\left(\begin{array}{ccc}
K^{\prime} & p & K \\
0 & 0 & 0
\end{array}\right) \\
& \times \iint R_{n^{\prime} l^{\prime}}\left(r_{e}\right) R_{n l}\left(r_{e}\right) R_{N^{\prime} K^{\prime}}^{\left(F^{\prime}, L^{\prime}\right)}\left(r_{h}\right) R_{N K}^{(F, L)}\left(r_{h}\right) \frac{r_{<}^{p+2}}{r_{>}^{p-1}} d r_{e} d r_{h} .
\end{aligned}
$$

In the above expression, due to the properties of the 3j-symbols, the summation on $p$ runs from $\max \left(\left|l-l^{\prime}\right|,\left|K-K^{\prime}\right|\right)$ to $\min \left(l+l^{\prime}, K+K^{\prime}\right)$.

* Electronic address: eariel@ff.oc.uh.cu

1 A. C. Albrecht, J. Chem. Phys. 34, 1476 (1961).

2 M. C. Klein, F. Hache, D. Ricard, and C. Flytzanis, Phys. Rev. B 42, 11123 (1990).

3 A. P. Alivisatos, T. D. Harris, P. J. Carrol, M. L. Steigerwald, and L. E. Brus, J. Chem. Phys. 90, 3463 (1989).

4 J. J. Shiang, S. H. Risbud, and P. Alivisatos, J. Chem. Phys. 98, 8432 (1993).

5 W. Jungnickel, F. Henneberger, and J. Plus, in Proceedings of the 22nd International Conference of Semiconductors, Vancouver, B.C., 1994, edited by D. J. Lockwood (World Scientific, Singapore, 1995), vol. III, p. 2011.

${ }^{6}$ G. Scamarcio, V. Spagnolo, G. Ventruti, M. Lugara, and G. C. Righini, Phys. Rev. B 53, R10489 (1996).

7 A. V. Baranov, S. Yamaguchi, and Y. Matsumoto, Phys. Rev. B 56, 10332 (1997).

${ }^{8}$ K. Inoue, K. Toba, A. Yamanaka, A. V. Baranov, A. A. Onushchenko, and A. V. Fedorov, Phys. Rev. B 54, R8321 (1996).

9 T. D. Krauss and F. K. Wise, Phys. Rev. B 55, 9860 (1997).

10 S. Nomura and T. Kobayashi, Phys. Rev. B 45, 1305 (1992).

11 Y. Chen, S. Huang, J. Yu, and Y. Chen, J. Lumin. 60\&61, 786 (1994).

12 J. C. Marini, B. Stebe, and E. Kartheuser, Phys. Rev. B 50, 14302 (1994).

13 M. G. Bawendi, P. J. Carroll, W. L. Wilson, and L. E. Brus, J. Chem. Phys. 96, 946 (1992).

14 E. P. Pokatilov, S. N. Klimin, V. M. Fomin, J. T. Devreese, and F. W. Wise, Phys. Rev. B 65, 075316 (2002), condmat/0111282.

15 M. P. Chamberlain, C. Trallero-Giner, and M. Cardona, Phys. Rev. B 51, 1680 (1995).

${ }^{16}$ R. Rodríguez-Suárez, E. Menéndez-Proupin, C. TralleroGiner, and M. Cardona, Phys. Rev. B 62, 11006 (2000).

17 A. V. Fedorov, A. V. Baranov, and K. Inoue, Phys. Rev. B 56, 7491 (1997).

18 E. Menéndez-Proupin, C. Trallero-Giner, and S. E. Ulloa,
Phys. Rev. B 60, 16747 (1999).

19 E. Menéndez-Proupin, C. Trallero-Giner, and A. GarcíaCristobal, Phys. Rev. B 60, 5513 (1999).

20 E. Menéndez, C. Trallero-Giner, and M. Cardona, Phys. Status Solidi B 199, 81 (1997).

21 E. Menéndez-Proupin, J. L. Peña, and C. Trallero-Giner, Semicond. Sci. Technol. 13, 871 (1998).

22 E. Menéndez-Proupin, C. Trallero-Giner, and A. GarcíaCristobal, Phys. Rev. B 60, 5513 (1999).

23 O. Madelung, ed., Landolt-Börnstein Numerical Data and Functional Relationships in Science and Technology, vol. III/22 (Springer, Berlin, 1986).

24 U. E. Laheld and G. T. Einevoll, Phys. Rev. B 55, 5184 (1997).

25 C. Hermann and C. Weisbuch, Phys. Rev. B 15, 823 (1977).

${ }^{26}$ In the case of valence band $\Gamma_{8}$ symmetry, the sum runs through heavy and hole bands, considering equal effective masses, which is necessary to obtain the symmetry of the Raman tensor. This is the only way to neglect band mixing consistently with the $\Gamma_{8}$ symmetry expressed in the elements $\mathbf{p}_{s_{z} j_{z} ; e \text {. }}$

27 A. Baldereschi and N. O. Lipari, Phys. Rev. B 8, 2697 (1973).

28 J.-B. Xia, Phys. Rev. B 40, 8500 (1989).

29 A. L. Efros, Phys. Rev. B 46, 7448 (1992).

30 J. D. Jackson, Classical Electrodynamics (Wiley, New York, 1962).

31 D. M. Brink and G. R. Satcher, Angular Momentum (Clarendon Press, Oxford, 1968).

32 M. G. Bawendi, W. L. Wilson, L. Rothberg, P. J. Carroll, T. Jedju, M. L. Steigerwald, and L. E. Brus, Phys. Rev. Lett. 65, 1623 (1990).

33 D. M. Mittleman, R. W. Schoenlein, J. J. Shiang, V. L. Colvin, A. P. Alivisatos, and C. V. Shank, Phys. Rev. B 49, 14435 (1994).

34 A. L. Efros, A. I. Ekimov, F. Kozlowski, and V. PetrovaKoch, Solid Stat. Commun. 78, 853 (1991).

35 C. Trallero-Giner and F. Comas, Phil. Mag. B 70, 583 
(1994). 